\title{
Sarcoma de Ewing extraósseo
}

\author{
Extraosseous Ewing's sarcoma
}

Ricardo Jorge da Silva Pereira',2, Ricardo Araújo², Sheila Cavalcante Pereira Araújoº, Miguel Rodrigues Lima Filho², Waleska Duarte Melo Albuquerque², Kátia Ramos Pereira Leite ${ }^{3}$, Vitor Engrácia Valenti ${ }^{4}$, Luiz Carlos de Abreu $^{4}$

\section{Resumo}

Introdução: 0 sarcoma de Ewing é um tipo de tumor que ocorre mais frequentemente nos ossos longos, podendo também estar presente na pelve, coluna e costelas. Admite-se que seja causado por uma anormalidade cromossômica, mais comumente entre os cromossomas 11 e 22 . Desenvolve-se a partir de um tipo de célula nervosa primitiva, podendo também ocorrer nas partes moles. É neoplasia óssea de comportamento biológico bastante agressivo, que acomete principalmente indivíduos abaixo dos 30 anos de idade e predomina discretamente em indivíduos do sexo masculino. A causa é desconhecida e não parece ser hereditária. São tumores extremamente raros em negros e asiáticos e o relato na população indígena é desconhecido. Relato do caso: 0 material coletado na biópsia foi enviado para exame imunoistoquímico, tendo sido utilizados dez anticorpos: desmina, mioglobina, actina, CD99, CD45, citoqueratina AE1/AE3, vimentina, sinaptolisina Ab-1, enolase neuroespecífica e proteína p53. 0 diagnóstico anatomopatológico foi de sarcoma indiferenciado de células pequenas com expressão difusa de CD99 e enolase neuroespecífica. 0 perfil imunoistoquímico foi consistente com o diagnóstico de sarcoma de Ewing/Tumor Neuroectodérmico Primitivo Periférico. Realizou-se a extirpação do tumor e o paciente encontra-se em tratamento de quimioterapia. Discussão: São tumores localmente agressivos com prognóstico reservado, geralmente com resultado terapêutico pobre.

Palavras-chave: Sarcoma; sarcoma de ewing; osso e ossos; neoplasias ósseas.

\begin{abstract}
Introduction: Ewing's sarcoma is a type of tumor which occurs frequently in long bones and it is also present in pelvis, spinal cord and ribs. It is thought to be caused by a chromosomal abnormality commonly between chromosomes 11 and 22 . It develops from a type of primitive nerve cell and it may also occur in soft tissues. It is a bone neoplasm which presents a very aggressive biologic profile; it affects mainly subjects younger than 30 years old and is slightly predominant in males. The cause is unknown and it does not seem to be hereditary. These tumors are extremely rare in blacks and Asians and the report in indigenous population is unknown. Case report: The material collected at biopsy was sent to immunohistochemical examination and we used ten antibodies: desmin, myoglobin, actin, CD99, CD45, cytokeratin AE1/AE3, vimentin, sinaptolisina Ab-1, neuron specific enolase and protein p53. The anatomopathological diagnosis indicated undifferentiated sarcoma of small cells with diffuse expression of CD99 and neuron-specific enolase. The immunohistochemical profile was consistent with the diagnosis of Ewing's sarcoma/Peripheral Primitive Neuroectodermal Tumor. The tumor was removed and the patient was in chemotherapy treatment. Discussion: These tumors are locally aggressive with a reserved prognosis, usually with poor therapeutic outcome.
\end{abstract}

Keywords: Sarcoma; sarcoma, ewing's; bone and bones; Bone neoplasm.

Recebido: $16 / 10 / 2009$

Revisado: $26 / 11 / 2009$

Aprovado: $28 / 1 / 2010$

\footnotetext{
Trabalho realizado no Centro de Ortopedia e Traumatologia, Maceió (AL), Brasil.

1 Disciplina de Ortopedia e Traumatologia da Faculdade de Medicina da Universidade Estadual de Ciências da Saúde de Alagoas (Uncisal), Maceió (AL), Brasil.

2 Centro de Ortopedia e Traumatologia, Maceió (AL), Brasil.

${ }^{3}$ Laboratório de Patologia Cirúrgica e Molecular do Hospital Sírio-Libanês, São Paulo (SP), Brasil.

${ }^{4}$ Faculdade de Medicina do ABC (FMABC), Santo André (SP), Brasil.

Endereço para correspondência: Ricardo Jorge da Silva Pereira - Departamento de Clínica Cirúrgica da Faculdade de Medicina da Universidade Estadual de Ciências da Saúde de Alagoas - Rua Doutor Jorge de Lima, 113 - Trapiche da Barra - CEP 57010-382 - Maceió (AL), Brasil - Tel: (82) 3315-6267 -

E-mail: ricardojsp@ig.com.br
} 


\section{Introdução}

O sarcoma de Ewing (SE) é um tipo de tumor que ocorre mais frequentemente nos ossos longos, podendo também estar presente na pelve, coluna e costelas. Admite-se que seja causado por uma anormalidade cromossômica, mais comumente entre os cromossomas 11 e 22 . Desenvolve-se a partir de um tipo de célula nervosa primitiva, podendo também ocorrer nas partes moles ${ }^{1,2}$.

A família de tumores de Ewing compreende um espectro de neoplasias de células neuroectodérmicas primitivas, as quais são células embrionárias que migram da crista neural. Esses tumores acometem primariamente osso e tecido mole. Dependendo do grau de diferenciação neural, são denominados “sarcoma de Ewing”, quando é um tumor indiferenciado, ou "Tumor Neuroectodérmico Primitivo Periférico" (PPNET, do inglês Peripheral Primitive Neuroectodermal Tumor), quando apresenta características de diferenciação neural. É uma neoplasia óssea de comportamento biológico bastante agressivo, que acomete principalmente indivíduos abaixo dos 30 anos de idade e predomina discretamente em indivíduos do sexo masculino² .

É o segundo tumor ósseo primário mais comum na infância. Atualmente, considera-se que é um tumor da linhagem do nervo parassimpático e compartilha de anormalidades específicas e genéticas moleculares com um tumor neuroectodérmico periférico mais diferenciado, nomeado de neuroepitelioma periférico ${ }^{3-6}$.

Em 1921, James Ewing observou que o "sarcoma ósseo de pequenas células" era, ao contrário do osteossarcoma, sensível ao implante de rádio. Na metade do século, o tratamento do SE empregava a cirurgia, a terapia local com radioterapia ou ambas. Mesmo quando o controle local era eficaz, mais de $90 \%$ dos pacientes morriam, geralmente por doença metastática ${ }^{3-9}$.

As experiências iniciais com quimioterapia iniciaram-se no início dos anos 60 do século passado e demonstraram que a quimioterapia adjuvante tem potencial de melhorar os índices de sobrevida e cura da doença ${ }^{3,9,10}$.

A causa é desconhecida e não parece ser hereditária. São tumores extremamente raros em negros e asiáticos; o relato na população indígena é desconhecido.

\section{Relato do caso}

E.R.S., masculino, índio, 24 anos de idade relatou que, há cinco meses, sentiu dor na região glútea, com irradiação para a face posterior do membro inferior esquerdo. Foi atendido pela clínica médica e encaminhado para um neurocirurgião, que solicitou uma tomografia computadorizada da região, cujo laudo foi normal. Foi medicado e, como a dor não cedeu, retornou ao serviço de origem após dois meses, tendo sido internado e medicado para melhora do quadro álgico.

Foi solicitada ultrassonografia pelo corpo clínico, mas esta não foi realizada pelo paciente. Continuou em tratamento medicamentoso para quadro álgico; porém, após mais dois meses, a dor se acentuou, sendo, então, encaminhado ao pronto-socorro de Maceió (AL). Foi submetido ao procedimento de drenagem de abscesso na região glútea e internado por três dias. Apresentou melhora clínica associada aos exames de imagem (ressonância nuclear magnética) sem alterações; recebeu alta, retornando a sua residência no interior de Alagoas.

Após um mês da alta hospitalar, retornou ao serviço de Ortopedia com queixa de quadro de lombociatalgia à esquerda. Nessa ocasião, apresentava tumoração na região glútea e lombar esquerda e cicatriz de mais ou menos $2,5 \mathrm{~cm}$ correspondente ao local da drenagem realizada.

Foi solicitado exame de imagem, sendo realizada ressonância nuclear magnética da região comprometida. O paciente retornou somente após um mês da realização do exame, por queixa de dor e dificuldades de deambulação, sendo internado no serviço de clínica médica. Nessa ocasião, com cinco meses de evolução do quadro clínico, manifestava lombociatalgia intensa, aliviada apenas com uso de morfina, bem como tumoração volumosa na região lombar e glútea, seguidas de febre e queda do estado geral.

Procedeu-se com a realização de tomografia computadorizada em caráter de urgência, disponível no interior do Estado, que evidenciou massas nas regiões glúteas e na pelve (Figura 1). Foi, então, imediatamente encaminhado ao centro cirúrgico, com possível diagnóstico de osteomielite do ilíaco e abscesso. Os achados operatórios mostraram uma massa esbranquiçada englobando os músculos da região glútea e pelve. Existia pouca quantidade de pus. O tecido estava organizado em
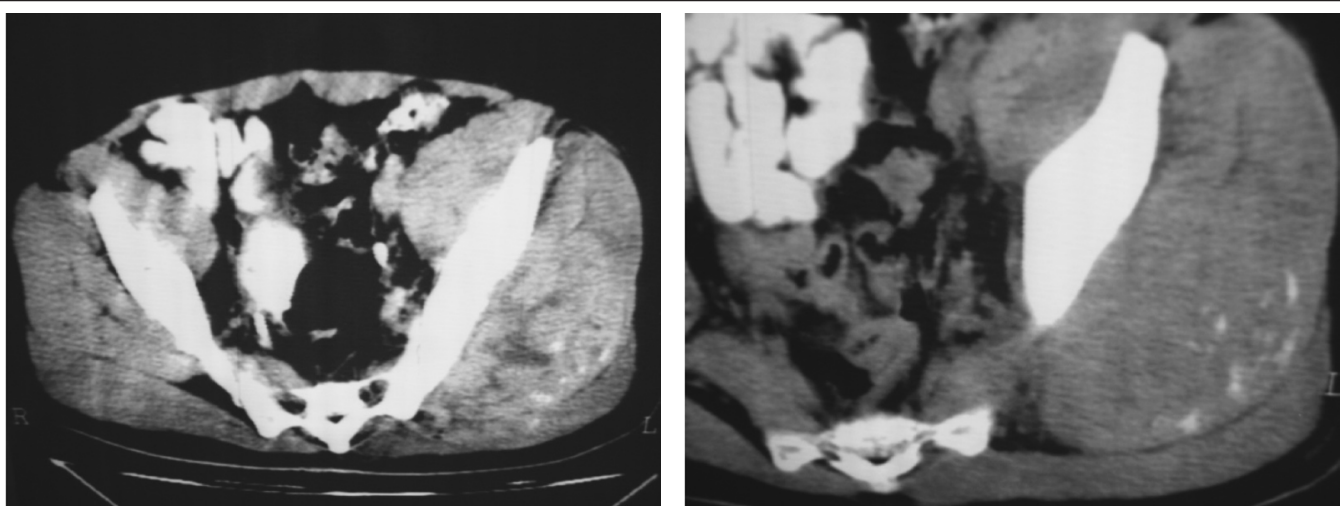

Figura 1 - Tomografia realizada no pré-operatório. As setas apontam para a tumoração intrapélvica e para a região glútea. Observa-se calcificação na tumoração. 
forma de fibras, com um aspecto de músculo exangue. O material retirado foi enviado para exame histopatológico e o diagnóstico foi de sarcoma indiferenciado de pequenas células com alto grau de malignidade.

Objetivando iniciar o tratamento, o material foi enviado para exame imunoistoquímico tendo sido utilizados dez anticorpos: desmina, mioglobina, actina, CD99, CD45, citoqueratina AE1/AE3, vimentina, sinaptolisina Ab-1, enolase neuroespecífica e proteína $\mathrm{p} 53$. O laudo emitido foi: "Sarcoma indiferenciado de células pequenas com expressão difusa de CD99 e enolase neuroespecífica. O perfil imunoistoquímico é consistente com o diagnóstico de sarcoma de Ewing/PNET”.

\section{Discussão}

O SE é um tipo de tumor de alta malignidade, que ocorre mais frequentemente nos ossos longos, podendo também estar presente na pelve, coluna, costelas e nas partes moles, sendo mais comum em crianças e adultos jovens ${ }^{1-6}$.

Angervall e Enzinger ${ }^{11}$ relataram os aspectos patológicos de 39 casos de sarcomas de pequenas células redondas ou ovais que comprometeram partes moles e os considerou histologicamente indistinguíveis do SE no osso. Relatou que a maioria comprometeu as extremidades inferiores e a região paravertebral.

Segundo Delattre et al..$^{12}$ o subgrupo de tumores de células pequenas redondas, identificados como pertencentes à família Ewing de sarcomas, pode ser definido de acordo com a lesão genética molecular específica, detectável por métodos eficientes.

Hadfield et al. ${ }^{13}$ descreveram diversos casos de sarcoma da família Ewing envolvendo estruturas do sistema nervoso, destacando-se o crânio, a medula espinhal, tecidos adjacentes, as meninges e o cérebro.

Hatori et al..$^{14}$ descreve que o SE, sarcoma de pequenas células redondas, cresce tanto nas partes moles como no osso, sendo um dos tumores de maior malignidade nas crianças e adultos jovens. Relata que poucos casos de sarcomas extraósseos são descritos na literatura, o que torna o difícil o exame dos achados biológicos desses tumores.

Perouli et al..$^{15}$ descreveu um caso de sarcoma extraósseo localizado na área paraespinhal torácica, com grande crescimento no período de um mês. Saxena et al. ${ }^{16}$ descreveu um caso na região do fígado.
Vários autores sugerem que o SE e os tumores primitivos neuroectodérmicos (TPNE) constituem um único grupo de tumores, no qual o SE seria a forma mais indiferenciada e o TPNE, a mais diferenciada. Essa afirmativa se baseia no fato de que ambos os tumores apresentam a translocação $t(11 ; 22)$ (q24;q12), encontrada em mais de $85 \%$ dos $\operatorname{casos}^{17}$.

De acordo com Jambhekar et al. ${ }^{18}$, os tumores da família Ewing apresentam dificuldade de diferenciação com outros tumores malignos de células pequenas e redondas; porém, mais de $90 \%$ têm EWS-FLI1, transcrição que age como um marcador potencial para diagnóstico molecular.

A semelhança histológica existente entre os tumores de células redondas pequenas torna-se um obstáculo para o diagnóstico ${ }^{19}$.

Atualmente, se aceita a existência da família Ewing de sarcomas, que inclui o SE extraesquelético e o tumor primitivo neuroectodérmico, os quais apresentam imunorreatividade para CD 99 e determinação antigênica pelo gene $\mathrm{MIC} 2^{20}$, de forma que, nos casos diagnosticados como sarcoma de células pequenas e redondas, devem-se efetivar esses exames determinantes da etiologia do sarcoma. Na maioria das vezes, o diagnóstico é realizado apenas por meio de exame histopatológico, insuficiente para o diagnóstico conclusivo, permanecendo sempre a dúvida sobre a origem desse tumor.

O CD99, identificado em aproximadamente todos os tumores da família dos SE, constitui-se num marcador positivo que pode ser utilizado como parte de uma bateria de exames imunoistoquímicos para o estabelecimento de diagnóstico diferencial entre os tumores de pequenas células redondas ${ }^{21}$.

Hasegawa et al..$^{22}$ diagnosticaram cinco casos de SE primários cutâneos que se apresentaram uniformemente imunorreativos à glicoproteína de superfície celular CD99.

CD99 é uma glicoproteína que apresenta um alto nível de expressão nas células do sistema hematopoiético, assim como nas células dos tumores de Ewing ${ }^{23}$. O CD 99 pode ser identificado em, aproximadamente, todos os tumores da família dos SE, constituindo um marcador positivo no estabelecimento de diagnóstico diferencial entre os tumores de pequenas células redondas.

Assim, pode-se concluir que o estudo imunoistoquímico é imprescindível na diferenciação e determinação do diagnóstico dos sarcomas da família Ewing.

\section{Referências}

1. Pereira RJS. Atlas de Ortopedia e Traumatologia Clínica. São Paulo: látria; 2006.

2. Catalan J, Fonte AC, Lusa JRB, Oliveira AD, Melo ES, Gonçalves CM. Sarcoma de Ewing: aspectos clínicos e radiográficos de 226 casos. Radiol. Bras. 2005;38(5):333-6.

3. Penna V, Lopes A, Tanaka MH, Wu TC, Melaragno R, Sidnei E. Sarcoma de Ewing: tratamento multidisciplinar. Rev. Bras. Ortop. 1993;28(11/12):809-12.

4. Bacci G, Picci P, Gitelis S, Borghi A, Campanacci M. The treatment of localized Ewing's sarcoma: The experience at the Instituto Ortopedico Rizzoli in 163 cases treated with and without adjuvant chemotherapy. Cancer. 1982;49(8):1561-1570.

5. Bader JL, Horowitz ME, Dewan R, Watkins E, Triche TJ, Tsokos M, et al. Intensive combined modality therapy of small round cell and undifferentiated sarcomas in children and young adults: local control and patterns of failure. Radiother Oncol. 1989;16(3):189-201.

6. Horowitz ME. Ewing's sarcoma: current status of diagnosis and treatment. Oncology (Williston Park). 1989;3(3):101-6. 
7. Butler MS, Robertson WW Jr, Rate W, D'Angio GJ, Drummond DS.Skeletal sequelae of radiation therapy for malignant childhood tumors. Clin Orthop Relat Res. 1990;(251):235-40.

8. Horowitz ME, Neff JR, Kun LE. Ewing's sarcoma: radiotherapy versus surgery for local control. Pediatr Clin North Am, 1991; 38(2):365-380.

9. Pritchard DJ. Small round cell tumors. In: Evarts CM editor. Surgery of the musculoskeletal tumor system. Rochester, NY: Churchill Livingstone; 1983. p.247-56.

10. Tepper J, Glaubiger D, Lichter A, Wackenhut J, Glatstein E. Local control of Ewing's sarcoma of bone with radiotherapy and combination chemotherapy. Cancer. 1980;46(9):1969-73

11. Angervall L, Enzinger FM. Extraskeletal neoplasm resembling Ewing's sarcoma. Cancer. 1975;36(1):240-51.

12. Delattre 0, Zucman J, Melot T, Garau XS, Zucker JM, Lenoir GM, et al. The Ewing family of tumors - a subgroup of small-round-cell tumors defined by specific chimeric transcripts. N Engl J Med. 1994;331(5):294-9,

13. Hadfield MG, Quezado MM, Williams RL, Luo VY. Ewing's family of tumors involving structures related to the central nervous system: a review. Pediatr Dev Patol. 2000;3(3):203-10

14. Hatori M, Doi H, Watanabe M, Sasano H, Hosaka M, Kotajima S, et al. Establishment and characterization of a clonal human extraskeletal Ewing's sarcoma cell line, EES1. Tohoku J Exp Med. 2006;210(3):221-30.

15. Perouli E, Chrysikopoulos H, Vlachos A, Koskinas A, Batistatou A, Polyzoidis $\mathrm{K}$. Imaging findings in paraspinal extra osseous Ewing sarcoma. JBR-BTR, 2006:89(6):310-2.
16. Saxena R, Sait S, Mhawech-Fauceglia P. Ewing sarcoma/primitive neuroectodermal tumor of yhe kidney: a case report. Diagnosed by immunohistochemistry and molecular analysis. Ann Diagn Pathol. 2006;10(6):363-6.

17. Pardini Jr A, Souza JMG. Clínica Ortopédica - tumores do sistema músculoesquelético. Rio de Janeiro: Editora Medsi; 2002.

18. Jambhekar N, Bagwan IN, Ghule P, Shet TM, Chinoy RF, Agarwal S, et al. Comparative analysis of routine histology, immunohistochemistry, reverse transcriptase polymerase chain reaction, and fluorescence in situ hybridization in diagnosis of Ewing family of tumors. Arch Pathol Lab Med. 2006;130(12):1813-8.

19. Lee C, Southey MC, Slater H, Auldist AW, Chow CW, Venter DJ. Primary cutaneous Ewing's sarcoma/peripheral primitive neuroectodermal tumors in childhood. A molecular, cytogenetic, and immunohistochemical study. Diagn Mol Pathol. 1995;4(3):174-81.

20. Isefuku S, Seki M, Tajino T, Hakozaki M, Asano S, Hojo H, et al. Ewing's sarcoma in the spinal nerve root: a case report and review of the literature. Tohoku J Exp Med. 2006;209(4):369-77.

21. Khoury JD. Ewing sarcoma family of tumors. Adv Anat Pathol. 2005;12(4):212-20.

22. Hasegawa S, Davison JM, Rutten A, Fletcher JA, Fletcher CD. Primary cutaneous Ewing's sarcoma: immunophenotypic and molecular cytogenetic evaluation of five cases. Am J Surg Pathol. 1998;22(3):310-8.

23. Scotlandi K, Baldini N, Cerisano V, Manara MC, Benini S, Serra M et al. CD99 engagement: an effective therapeutic strategy for Ewing tumors. Cancer Res. 2000;60(18):5134-42. 